\title{
Portación de levaduras en manos de estudiantes de las carreras de Medicina y Enfermería de la Universidad de Talca, Chile
}

\author{
(Carriage of yeasts in the hands of the students of medicine and nursing \\ careers at the University of Talca, Chile)
}

\author{
Ardaia Muango Chinduma ${ }^{1 *}$, Pedro Brevis Azócar ${ }^{1}$, Claudia Mora Pareja ${ }^{1}$ \\ ${ }^{1}$ Departamento de Microbiología, Universidad de Talca, Chile. \\ *Autor para correspondencia: ardaiatomas794@gmail.com
}

RECIBIDO: 19 de Octubre de 2017

APROBADO: 17 de Noviembre de 2017

DOI: 10.22370/bolmicol.2017.32.2.994

LOS AUTORES DECLARAN NO TENER CONFLICTO DE INTERESES

Palabras claves: Colonizacion en manos, identificación de levaduras, lavado de manos.

Key words: Colonization in hands, yeast identification, hand washing.

\section{RESUMEN}

Introducción: En las últimas décadas, la frecuencia de infecciones fúngicas invasivas causadas por levaduras ha aumentado en las Unidades de Cuidados Intensivos (UCI), especialmente en pacientes graves con estadías prolongadas y en inmunocomprometidos. En ocasiones pueden presentarse como infecciones asociadas a la atención de salud (IAAS) por incumplimiento de medidas efectivas en prevención. Candida albicans es la especie más frecuentemente aislada, aunque el número de especies no albicans resistentes a fluconazol ha aumentado y la mortalidad asociada es mayor en los pacientes infectados con estas especies, motivo de preocupación puesto de manifiesto por los datos de vigilancia recientes. Objetivo: establecer la frecuencia de portación de levaduras de importancia clínica aisladas de las manos de estudiantes de las carreras de Medicina y Enfermería de la Universidad de Talca. Métodos: se tomaron 208 muestras de las manos de estudiantes, estas se dividieron en dos categorías: la primera es el ciclo básico de los estudiantes sin contacto con hospitales, la segunda es el pre-clínico y clínico de los estudiantes que tienen contacto con hospitales. Resultados: hubo $11.2 \%$ de portación en manos de estudiantes de Medicina y $9.9 \%$ para los de Enfermería. En los aislamientos predominó la Candida parapsilosis (45.5\%); Candida guillermondii (18.2\%); Candida famata (9.1\%), y Candida albicans (4.5\%) Schwanniomyces etchellsii (18.2\%) y Cryptococcus humícola $(4.5 \%)$. Conclusión: la portación de levaduras en manos de estudiantes de carreras de salud, aumenta significativamente en aquellos que tienen mayor contacto con las unidades hospitalarias, así como la diversidad de especies y la cantidad de unidades formadoras de colonias. 
Portación de levaduras en manos de estudiantes - Muango A. et al

\begin{abstract}
Introduction: In the last decades, the frequency of invasive fungal infections caused by yeast has increased in the Intensive Care Units (ICU), especially in severe patients with prolonged stays and in immunocompromised patients. Sometimes they can present as infections associated with health care (IAAS) due to non-compliance with effective prevention measures. Candida albicans is the most frequently isolated species, although the number of non-albicans species resistant to fluconazole has increased and the associated mortality is higher in patients infected with these species, a concern highlighted by recent surveillance data. Objective: to establish the frequency of carrying of yeasts of clinical importance isolated from the hands of students of the careers of Medicine and Nursing of the University of Talca. Methods: 208 samples were taken from the hands of students, these were divided into two categories: the first is the basic cycle of students without contact with hospitals, the second is the pre-clinical and clinical students who have contact with hospitals. Results: there was a: $11.2 \%$ portation in the hands of medical students and $9.9 \%$ for Nursing students. In the isolates Candida parapsilosis prevailed (45.5\%); Candida guillermondii (18.2\%); Candida famata (9.1\%), and Candida albicans (4.5\%) Schwanniomyces etchellsii (18.2\%) and Cryptococcus humicola $(4.5 \%)$. Conclusion: the carrying of yeasts in the hands of students of health careers increases significantly in those who have greater contact with hospital units, as well as the diversity of species and the number of colony forming units.
\end{abstract}

\section{INTRODUCCIÓN}

Las infecciones por Candida spp. constituyen la cuarta causa de infecciones del torrente sanguíneo de origen nosocomial y presentan una elevada mortalidad (30-50\%). La especie más frecuentemente aislada es $C$. albicans, sin embargo, en la última década se ha observado un aumento de otras especies de Candida "no albicans", destacando una mayor frecuencia de C. parapsilosis (1, $2)$. En seres humanos, C. albicans puede causar varios tipos de infecciones de gravedad variable, que pueden ir desde candidiasis oral o vaginal hasta enfermedad invasora con compromiso de órganos $(3,4,5)$. Los principales factores de riesgo incluyen catéteres venosos centrales, nutrición parenteral que permiten el acceso directo del hongo al torrente sanguíneo, la aplicación de antibacterianos de amplio espectro, que permiten el crecimiento excesivo de hongos y trauma o cirugía gastrointestinal, que interrumpe las barreras de las mucosas $(6,7,8)$. Entre los factores de virulencia atribuidos a $C$. albicans destaca su potencial capacidad de formación de biofilm y persistencia en el hospedero en forma de reservorios (colonización del tracto urogenital y vías respiratorias) (9).

Existe evidencia de transmisión horizontal de levaduras intrahospitalaria y que se ha relacionado a portación en las manos de personal de salud $(3,10)$. Estudios han demostrado que la portación de levaduras en las manos de personas sanas es inferior a 5\%, sin embargo en trabajadores de la salud puede variar entre $20 \%$ en el personal Médico y $80 \%$ en el de Enfermería (11).

El objetivo de este trabajo fue establecer la frecuencia de portación de levaduras de importancia clínica de la división Ascomycota y Basidiomycota aisladas en manos de estudiantes de las carreras de Medicina y Enfermería de la Universidad de Talca.

\section{MATERIAL Y METODOS}

Estudio descriptivo realizado entre junio y agosto de 2017. Fueron incluídos un total de 208 
alumnos, los cuales fueron divididos para el análisis en 2 grupos "ciclo básico" $(\mathrm{n}=116)$ y "preclínico / clínico ” $(\mathrm{n}=92)$.

Los integrantes del grupo básico eran de segundo año de medicina $(n=77)$ o de cursos iniciales de la carrera de enfermería $(n=39)$, ninguno con actividades ni contacto con centros hospitalarios. El grupo "pre-clínico / clínico” estaba constituido por estudiantes de Medicina de cuarto a séptimo año $(n=40)$ y de tercer a quinto año de Enfermería $(\mathrm{n}=52)$, ambos con contacto a tiempo parcial o permanente con centros hospitalarios durante el período de estudio.

Obtención de muestras

La toma de muestras se realizó con torundas embebidas con suero fisiológico estéril a $0.9 \%$. Se aplicó $0,5 \mathrm{ml}$ en las palmas de las manos de los estudiantes y luego las frotaron durante 30 segundos simulando un lavado de las palmas de las manos, dorso, pliegues, interdigital y uñas; en seguida se recolectó la muestra con una torunda estéril, que fue colocada en un tubo de vidrio estéril conteniendo $0,5 \mathrm{ml}$ de suero fisiológico estéril.

Procesamiento de las muestras

Las muestras se inocularon en agar glucosado Sabouraud (BBL) suplementado con cloranfenicol $(500 \mathrm{mg} / \mathrm{L})$, abarcando toda la superficie del medio, incubándose a $35^{\circ} \mathrm{C}$ durante 7 días. También se realizó siembra en el medio CHROMagar Candida (bioMérieux) y las placas se incubaron a $35^{\circ} \mathrm{C}$ hasta 7 días.

Interpretación del cultivo:

Para la confirmación del crecimiento de colonias sospechosas el cultivo se observó diariamente y la presencia de levaduras se corroboró microscópicamente. La cantidad de unidades formadoras de colonias se evaluó de forma semicuantitativa, agrupando el número de colonias según una escala arbitraria creada para la investigación, donde la ausencia de colonias se evaluó como negativo (-), el crecimiento entre 1 y 5 UFC como débil $(+)$, de 6 a 20 UFC como moderado $(++)$ y sobre 20 UFC como abundante $(+++)$.

Tras analizar las características de las colonias en agar Sabouraud, que presentaban colonias blancas y lisas (compatibles con Candida spp.), se realizaron las siguientes pruebas: formación de tubo germinal, micromorfología en agar maíz con Tween 80 (1\%) y auxonograma usando glucosa, celobiosa, dulcitol, galactosa, inositol, L-arabinosa, lactosa, maltosa, manitol, melibiosa, rafinosa, ramnosa, sacarosa, trehalosa y xilosa. En el medio CHROMagar Candida crecieron colonias verdes esmeralda que correspondieron a C. albicans y otras colonias de color blanco que fueron identificadas por API-32C bioMérieux como $C$. parapsilosis, C. guillermondii, C. famata, C. albicans, Cryptococcus humícola y Schwanniomyces etchellsii. Las cepas de Candida identificadas por este sistema dieron un margen de seguridad de $99.9 \%$ y las especies Schwanniomyces etchellsii y Cryptococcus humicola dieron un porcentaje más bajo $85-89 \%$, por lo cual se deberían realizar otras pruebas adicionales para una identificación definitiva.

Análisis estadístico. Se utilizó la prueba de chi-cuadrado (x2) para evaluar si existe asociación entre los grupos pre-clínicos y clínicos (variable independiente) y la frecuencia de colonización por levaduras (variable dependiente). Se consideró significativo un valor $\mathrm{p}<0,05$. El análisis de los datos fue realizado con el programa XYZ versión $\mathrm{ABC}$.

\section{RESULTADOS}

Se aislaron levaduras en las manos de 13 estudiantes de Medicina (11.2\%) y de 9 de Enfer- 
mería (9.8\%). Se observó un aumento de la prevalencia de portación, directamente relacionada con el ciclo de estudio y el tiempo de contacto de los estudiantes con las unidades hospitalarias, siendo para el ciclo básico $1.7 \%$ y $10.3 \%$ pre-clínico / clínico. Resultados similares fueron observados en los participantes de Enfermeria con portación de $3.3 \%$ y $6,6 \%$ para los grupos ciclo básico y preclínico / clínico respectivamente. Estas diferencias fueron estadísticamente significativas $(p<0,05)$ (Tabla 1). La observación y cuantificación de co- lonias para los estudiantes portadores de levaduras fue bastante variable; la mayoría presentó entre 1 y 7 UFC por placa. Se observó predominio de especies del género Candida (77.3\%), seguida de las especies Schwanniomyces etchellsii (18.2\%) y Cryptococcus humícola (4.5\%). Las especies del género Candida se distribuyeron con una frecuencia de C. parapsilosis con 10 aislamientos (45.5\%), C. guillermondii con 4 aislamientos $(18.2 \%), C$. famata con 2 aislamientos $(9.1 \%)$, y hubo sólo 1 aislamiento de C. albicans (4.5\%) (Tabla 2).

Tabla 1. Identificación por grupos de estudiantes portadores y no portadores de levaduras en sus manos.

\begin{tabular}{|c|c|c|c|c|}
\hline & \multicolumn{2}{|c|}{ Medicina } & \multicolumn{2}{c|}{ Enfermería } \\
\hline Muestras procesadas & Portadores & Porcentaje & Portadores & Porcentaje \\
Ciclo básico & 2 & $1.7 \%$ & 3 & $3.3 \%$ \\
Pre clínico /clínico & 11 & $9.5 \%$ & 6 & $6.6 \%$ \\
\hline Total portadores & 13 & $11.2 \%$ & 9 & $9.9 \%$ \\
\hline Muestras procesadas & NO portadores & Porcentaje & NO portadores & Porcentaje \\
Ciclo básico & 75 & $64 \%$ & 36 & $39.6 \%$ \\
\hline Pre clínico /clínico & 29 & $24 \%$ & 46 & $50.5 \%$ \\
\hline Total no portadores & 104 & $88 \%$ & 82 & $90.1 \%$ \\
\hline Total general & 116 & $100 \%$ & 91 & $100 \%$ \\
\hline
\end{tabular}

208 número total de alumnos estudiados por grupos en las respectivas carreras Ciclo básico vs. Pre-clínico y clínico para Medicina $(\mathrm{p}=0.0001) * * *$ Ciclo básico vs. Pre-clínico y clínico para Enfermería $(\mathrm{p}=0.7273)$ * 
Portación de levaduras en manos de estudiantes - Muango A. et al

Tabla 2. Cuantificación de especies de levaduras aisladas durante el estudio.

\begin{tabular}{|c|c|c|c|c|c|}
\hline \multirow[b]{2}{*}{ Especies } & \multicolumn{2}{|l|}{ Medicina } & \multicolumn{3}{|l|}{ Enfermería } \\
\hline & $\begin{array}{c}\text { N de aislamientos } \\
\text { Ciclo básico }\end{array}$ & $\begin{array}{l}\mathrm{N} \text { de aislamientos } \\
\text { pré-clínico } \\
\text { clínico/clínico }\end{array}$ & $\begin{array}{l}\mathrm{N} \text { de aislamientos } \\
\text { Ciclo básico }\end{array}$ & $\begin{array}{l}\text { N de aislamientos } \\
\text { Pre clínico } \\
\text { /clínico }\end{array}$ & Porcentaje \\
\hline C. parapsilosis & 0 & 5 & 1 & 4 & $45.5 \%$ \\
\hline C. guilliermondii & 0 & 2 & 2 & 0 & $18.2 \%$ \\
\hline C. famata & 0 & 2 & 0 & 0 & $9.1 \%$ \\
\hline C. albicans & 0 & 0 & 1 & 0 & $4.5 \%$ \\
\hline $\begin{array}{l}\text { Schwanniomyces } \\
\text { etchellsii }\end{array}$ & $\begin{array}{l}2 \\
0\end{array}$ & $\begin{array}{l}1 \\
1\end{array}$ & $\begin{array}{l}0 \\
0\end{array}$ & $\begin{array}{l}1 \\
0\end{array}$ & $\begin{array}{r}18.2 \% \\
4.5 \%\end{array}$ \\
\hline Cryptococcus humicola & & & & & \\
\hline Total & 2 & 11 & 4 & 5 & $100 \%$ \\
\hline
\end{tabular}

\section{DISCUSIÓN}

El uso creciente y la necesidad de dispositivos médicos implantados y catéteres venosos centrales en el manejo del cuidado del paciente son razones importante por las cuales la incidencia de infecciones por Candida spp. han aumentado constantemente $(4,12)$. Se considera que las manos cumplen un papel importante como vehículo de transmisión exógena de levaduras hacia el paciente en las unidades hospitalarias. La presencia de levaduras en las manos del personal de la salud ha sido ampliamente documentada (13-15). En el presente estudio, la portación de levaduras en manos fue de $11.2 \%$ para los estudiantes de la carrera de Medicina y $9.9 \%$ para la carrera de Enfermería. Las 22 cepas de levaduras aisladas de las manos de estudiantes fueron identificadas bioquímicamente como C. parapsilosis, C. guilliermondii, C. famata, C. albicans, Schwanniomyces etchellsii y Cryptococcus humícola. Estos datos son cercanos a los obtenidos por Cleveland et al.
(16), en cuanto a las especies aisladas. Estudios basados en la población en dos regiones Metropolitanas de los Estados Unidos entre 2008-2013, demostraron que Candida albicans fue la especie más común (1.401 casos, 36\%), seguida de Candida glabrata (27\%), C. parapsilosis (15\%), y finalmente $C$. tropicalis (6\%). En los países latinoamericanos la mayor incidencia de Candidemia se describe en Colombia y la más baja en Chile; C. albicans es la especie más frecuentemente notificada seguida de C. parapsilosis y C. tropicalis (17). En Chile en un estudio realizado en cinco hospitales de Valparaíso, predominó C. albicans, seguida de C. tropicalis y C. parapsilosis (18). En nuestro trabajo se observó una portación importante de levaduras con predominio de Candida spp (77\%). Se aislaron 10 cepas de C. parapsilosis (45.5\%), 4 cepas de C. guillermondii (18.2\%), 2 cepas de $C$. famata $(9.1 \%)$, y finalmente una cepa de $C$ albicans (4.5\%). Otras especies de levaduras fueron 4 cepas de Schwanniomyces etchellsii (18.2\%) y una cepa de Cryptococcus humicola 
Portación de levaduras en manos de estudiantes - Muango A. et al

(4.5\%). Estos resultados fueron similares a otros estudios reportados $\left.{ }^{13,19,20}\right)$. Es importante, destacar que $C$. parapsilosis se aisló en un alto porcentaje en las manos de estudiantes de pre-clínico y clínico de Medicina y Enfermería.

Actualmente, C. parapsilosis es considerado un hongo patógeno importante, siendo la especie de Candida predominante en las infecciones del torrente sanguíneo, es la principal especie responsable de brotes de fungemia nosocomial, especialmente en Unidades de Cuidados Intensivos neonatales (UCI) (21). En Chile, $\mathbf{C}$. parapsilosis es considerado un agente emergente, asociado a CVC y catéteres de HDL (18). Estudios realizado en Costa Rica entre los años 2007 y 2011 revelaron que $C$. parapsilosis fue la especie predominante en cuatro de los 5 años estudiados seguido de $C$. albicans (22). En el entorno clínico esta levadura oportunista se ha asociado con las manos de trabajadores del área de salud, y es particularmente frecuente en infecciones sistémicas en neonatos prematuros de bajo peso, pacientes cateterizados, además de sujetos bajo esquemas de hiperalimentación intravenosa (23). Schwanniomyces etchellsii es una levadura reconocida como de baja patogenicidad, se describió por primera vez el año 2016 un caso de colecistitis humana causada por este agente (24). Cryptococcus humicola es una levadura basidiomycete que ha sido aislada de varios substratos como plantas, suelo y especímenes clínicos (25).

El resultado del presente trabajo demuestra que la portación de levaduras en las manos de estudiantes de carreras de salud, aumenta significativamente en aquellos que tienen mayor contacto con las unidades hospitalarias, así como la diversidad de especies y la cantidad de unidades formadoras de colonias, por lo tanto, es indispensable educar correctamente a los estudiantes sobre el procedimiento e importancia del lavado de manos en la prevención y control de la transmisión cruzada de estos patógenos.

\section{REFERENCIAS}

1. Horn DL, Neofytos D, Anaissie EJ, Fishman JA, Steinbach WJ, Olyaei AJ, et al. Epidemiology and outcomes of candidemia in 2019 patients: data from the prospective antifungal therapy alliance registry. Clin Infect Dis. 2009;48(12):1695-703.

\section{Nucci M, Queiroz-Telles F, Alvarado-Matute} T, Tiraboschi IN, Cortes J, Zurita J, et al. Epidemiology of Candidemia in Latin America: A Laboratory-Based Survey. PLoS One. 2013;8(3):

3. Cleveland A., Harrison Lee H., Farley MM., et ál. Declining Incidence of Candidemia and the Shifting Epidemiology of Candida Resistance in Two US Metropolitan Areas, 2008-2013: Results from Population-Based Surveillance. Plos One. 2015; 10(3)

4. Tsui C, Kong EF, Jabra-Rizk MA. Pathoge- nesis of Candida albicans biofilm. Pathog Dis. 2016;74(4):ftw018.

5. Picazo JJ, González-Romo F, Candel FJ. Candidemia in the critically ill patient. International Journal of Antimicrobial Age 2008;32.

6. Pappas PG, Alexander BD, Andes DR, Hadley S, Kauffman CA, Freifeld A, et al. Invasive Fungal Infections among Organ Transplant Recipients: Results of the Transplant-Associated Infection Surveillance Network (TRANSNET). Clinical Infectious Diseases. 2010;50(8):1101-11.

7. Mayer FL, Wilson D, Hube B. Candida albicans pathogenicity mechanisms. Virulence. 2013;4(2):119-128.

8. Ortega M, Marco F, Soriano A, Almela M, 
Martinez JA, Lopez J, et al. Candida species bloodstream infection: epidemiology and outcome in a single institution from 1991 to 2008 . J Hosp Infect. 2011;77(2):157-61.

9. Jabra-Rizk MA, Kon g EF, Tsui C, Nguyen MH, Clancy CJ, Fidel PL, et al. Candida albicans Pathogenesis: Fitting within the Host-Microbe Damage Response Framework. Infect Immun. 842016. p. 2724-39.

10. Sanchez-Barrena MJ, Martinez-Ripoll M, Galvez A, Valdivia E, Maqueda M, Cruz V, et al. Structure of bacteriocin AS-48: from soluble state to membrane bound state. J Mol Biol. 2003;334(3):541-9.

11. Tsui C, Kong EF, et al. Pathogenesis of Candida albicans biofilm. Pathogens and Disease. 2017;74(4).

12. Tournu H, Van Dijck P. Candida biofilms and the host: models and new concepts for eradication. Int J Microbiol. 2012;2012:845352.

13. Saúl García Y, Francisco de Miranda, Hernández Valles R. Aislamiento de Candida spp. en ambiente y personal que labora en una unidad de cuidados intensivos. Rev Soc Ven Microbiol. 2014;34(1):27-32.

14. Silva V, Zepeda G, Rybak ME, Febre N. Yeast carriage on the hands of Medicine students. Rev Iberoam Micol. 2003;20(2):41-5.

15. Wang H, Zhang L, Kudinha T, Kong F, Ma XJ, Chu YZ, et al. Investigation of an unrecognized large-scale outbreak of Candida parapsilosis sensu stricto fungaemia in a tertiary-care hospital in China. Sci Rep. 62016.

16. Cleveland AA, Harrison LH, Farley MM, Hollick R, Stein B, Chiller TM, et al. Declining incidence of candidemia and the shifting epidemiology of Candida resistance in two US metropolitan areas, 2008-2013: results from population-based surveillance. PLoS One. 2015;10(3):e0120452.

17. Nucci M., Queiroz-Telles F., Alvarado-Matute T., Tiraboschi I., Cortes J., Zurita J., et al. Epidemiology of Candidemia in Latin America: A Laboratory-Based Survey. Plos One. 2013; 8(3)

18. Cruz Ch R, Piontelli L E. Enfermedad fúngica invasora en pacientes de cinco hospitales de la Región de Valparaíso, Chile: 2004 a 2009. Rev chil infectol. 2011;28(2):123-9.

19. Farooqi JQ, Jabeen K, Saeed N, Iqbal N, Malik B, Lockhart SR, et al. Invasive candidiasis in Pakistan: clinical characteristics, species distribution and antifungal susceptibility. J Med Microbiol. 2013;62(Pt 2):259-68.

20. Bonassoli LA, Bertoli M, Svidzinski TI. High. frequency of Candida parapsilosis on the hands of healthy hosts. J Hosp Infect. 2005;59(2):159-62.

21. Kong F, Zou G-L, Wang H, Lu J, Liao K, Zhang L, et al. Investigation of an unrecognized large-scale outbreak of. Scientific Reports. 2016:6.

22. Villalobos JM, Castro JA, Avilés A, et al. Candida parapsilosis: principal causa de candidemia en un hospital de referencia para adultos de Costa Rica. Rev chil infectol. 2016;33(2):159-65.

23. Treviño-Rangel R., Gonzalez-Gonzalez J., Garza-Gonzalez E., Gonzalez G. Candida parapsilosis, una amenaza desafiante. Medicina Universitaria. 2012; 14(56):157-165

24. Relich RF, Schmitt BH, Koehlinger J, Wiederhold NP, May M. Schwanniomyces etchellsii: an unusual cause of fungemia in a patient with cholecystitis. Diagnostic microbiology and infectious disease. 2016;84(3):221-2.

25. Takashima M, Sugita T, Shinoda T, Nakase T. Reclassification of the Cryptococcus humicola complex. Int J Syst Evol Microbiol. 2001;51(Pt 6):2199-210. 